\title{
High Order UWB Pulses Generation based on a Scalable Phase-to-intensity Technique
}

\begin{abstract}
In this work, a scheme that relies on phase-to-intensity (PM-IM) conversion for high order UWB pulses generation is proposed and demonstrated by using a customized optical filter. The obtained triplet pulse reveals high efficiency and proper fitting for FCC standards.
\end{abstract}

\section{Introduction}

Current trends in wireless networks technologies demand low complexity, low cost, low power consumption and high data-rate connectivity within the operational space. Under this background, ultra wideband (UWB) has emerged as an interesting topic of research supported for its capacity of sharing existing radio spectrum resources rather than demanding new spectral bands ${ }^{1}$. Nevertheless, due to the low power spectral density (PSD) value of $\leq 41.3 \mathrm{~dB} / \mathrm{MHz}$ of the transmitted signal settled by FCC regulations ${ }^{2}$, there is a trade-off between data rate and communication distance.

Several techniques to generate modulate and distribute UWB pulses have been proposed $^{3}$, not only to increase the scope of coverage area but also to provide undisrupted service across different networks. Generating directly in the optical domain avoids any extra electrical to optical conversion. In addition, the combination of UWB and Microwave photonics (MWP) techniques offers numerous beneficial features such as light weight, small size, tunability and immunity to electromagnetic interference ${ }^{4}$. Among the standard UWB pulses employed in modern literature, we can find monocycles and doublets. However, these pulses adapt poorly to the FCC spectral mask ${ }^{5}$. In this context, generation of high-order pulses stands as a fundamental niche to fully comply FCC regulations.

Different MWP techniques for UWB generation of low and high order pulses have been reported in the last years including solutions based on optical spectral shaping and dispersion-induced frequency-to-time mapping ${ }^{6}$, microwave photonic filtering and SOA nonlinear operation $^{8}$. All these proposals even though effective do not represent entirely optimized schemes, since for each Gaussian base pulse generated only one coefficient is inserted to the system and into the generated waveform. In this way, an alternative technique was recently proposed for obtaining UWB high order signals consisting on the merge of various low order UWB pulses, such as monocycles and doublets to create triplets or quadruplets ${ }^{9,10}$.

In this letter, we present a proposal for generation of UWB high order pulses, which is focused on the merge of various low order UWB pulses. The principle of operation relies on the combination of a phase modulator with a customized FBG array for a simultaneous process of PM-IM conversion and pulse shaping. The notion of combining phase modulation with optical filters has been approached previously ${ }^{11,12}$. However, high order pulses have not been experimentally reported with this promising technique. In the first one ${ }^{11}$, the PM-IM conversion takes place in a single FBG, constraining any possibility of high-order pulses generation. In the other one ${ }^{12}$, the design shows a complex receiver due to the presence of a balanced photodetector (BPD). Therefore, the bulky architecture limits the possibilities of an integrated optics approach. The remaining of the paper is organized as follows: firstly, the operation principles is presented and analysed. Secondly, experimental measurements of the generated UWB pulse, in both time and frequency domain, are presented revealing efficiency and a proper fit in terms of FCC settled standards. Apart from the generation of pulses with an efficient compliance of the FCC spectral mask, this optical UWB system holds the possibility of pulse codification, implementing different modulation formats such as Pulse Position Modulation (PPM) and $\mathrm{Bi}$ Phase Modulation (BPM), which will be addressed at the end of the experimental section. Finally, conclusions are addressed. 


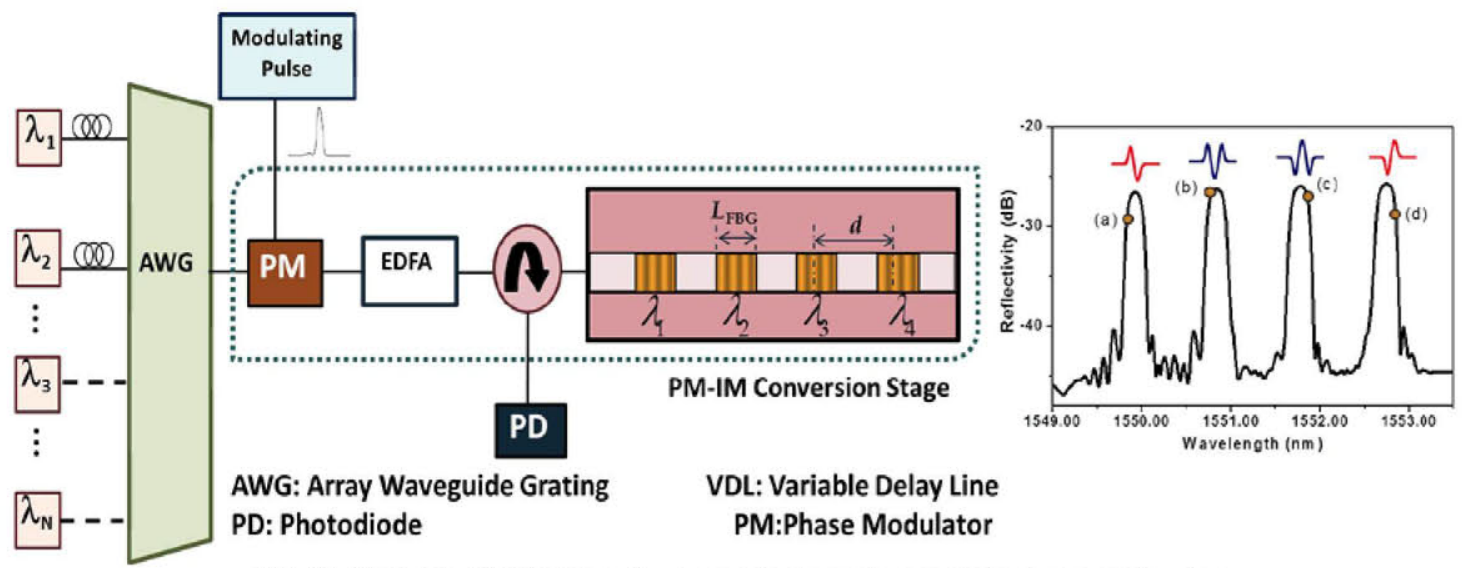

Fig. 1: Scalable UWB high order generator based on a photonic superstructure.

\section{Principle of Operation}

Figure 1 depicts in detailed the proposed photonic architecture. In the first contemplated segment, light from a set of $\mathrm{N}$ optical sources is launched to a phase modulator (PM) by means an array waveguide grating (AWG). The PM is modulated by Gaussian like pulses coming from an electrical pulse generator providing an OOK data sequence to be transmitted. In this implementation, we expand the concept of PMIM conversion with a single fiber Bragg grating (FBG) operating as a frequency discriminator ${ }^{13}$ by introducing high order pulses generation and modulation capacities through a FBG superstructure. In this context, by locating the optical carrier at the linear or the quadrature slope of the FBG reflection spectrum, the base monocycle or doublet pulses can be obtained. Deriving in operational points " $A$ " (positive monocycle), "B" (positive doublet) "C" (negative doublet) and "D" (positive monocycle).

The design of a suitable FBG array is a significant matter since the quality of the reflected spectrum and the distance between each one will determine the quality of the highorder pulse to be produced. First of all, when designing the array, we had to consider a specific apodized profile to reduce the secondary lobes of the FBG spectrum in order to minimize the crosstalk between channels. Secondly, there is the separation factor $d$, this parameter holds a direct relation with the optical delay between taps $\left(\Delta \tau=2 n_{0} d / c\right)$. In order to be working with a FSR/2 around $6.45 \mathrm{GHz}$, in agreement with the central frequency of the UWB spectral band we require a FSR $(1 / \tau)$ of approximately $12.9 \mathrm{GHz}$ and an optical delay $(\Delta \tau)$ of $77 \mathrm{ps}$. Therefore, $d$ must hold a value around to $8 \mathrm{~mm}$. Finally, there is the length factor I. A $7 \mathrm{~mm}$ length is considered an optimal value for each FBG within the array in order to avoid overlapping between adjacent gratings in the fabrication process. In this specific solution, the phase modulated pulse is introduced to a FBG based superstructure through an optical circulator which will operate in function of the optical wavelength selected for each optical source.

\section{Experimental Results}

To demonstrate and validate the capabilities of generation in the scheme of Fig. 1, the generation of UWB high order pulses was accomplished by combining several low-order signals. For this experiment, we employed two lasers with an optical power value of $5.5 \mathrm{dBm}$ each and wavelengths located at $1549.837 \mathrm{~nm}$ and $1550.683 \mathrm{~nm}$. Activation of such optical sources is proportional to the number of lower order pulses to be reconfigured in the outcome signal and their values are directly related with the base UWB waveforms to be employed and hence the PM-IM conversion working point. In this case the triplet was accomplished by means of the lineal sum of two inverted doublet pulses, corresponding to the " $\mathrm{C}$ " and " $\mathrm{B}$ " operational points. Fig. 2(a) exhibits the obtained UWB triplet waveform, where the blue line corresponds to the theoretical simulation and Fig. 2(b) plots its corresponding spectral representation.


Fig. 2: (a) Generated UWB (b) corresponding spectrum. FCC mask is plotted in red line. 
In order to maximize the functionalities of the proposed system, along with high-order waveforms generation, implementation of diverse modulation formats such as Pulse Position Modulation (PPM) and Bi-Phase Modulation (BPM) is also feasible. In the case of PPM modulation, it can be achieved by employing the entire FBGs array and tuning the proper wavelength in each FBG. Such wavelengths can be tagged as $\lambda_{1 \mathrm{~A}}, \lambda_{2 \mathrm{~A}}, \lambda_{3 \mathrm{~A}}$ and $\lambda_{4 \mathrm{~A}}$ with corresponding values of $1549.721 \mathrm{~nm}$, $1550.631 \mathrm{~nm}, 1551.568 \mathrm{~nm}$ and $1552.561 \mathrm{~nm}$. Figure 3(a) depicts the generated pulses for PPM modulation, with a separation between the original pulse and the delayed ones of approximately $77 \mathrm{ps}, 154 \mathrm{ps}$ and $231 \mathrm{ps}$. In the case of BPM, this format was achieved by the juxtaposition of two inverted pulses, setting up one FBG and two optical sources. The FBG1 works as the optical filter for this measurement with a Bragg wavelength of $1549.808 \mathrm{~nm}$. The first tuned configuration corresponds to the " $A$ " working point, which produces a positive monocycle and the inverted pulse is related to the "D" working point and thus to a negative monocycle. Figure $3(\mathrm{~b})$ shows the two low-order UWB pulses obtained for BPM by switching the optical wavelength around values $\lambda_{1 \mathrm{~A}}=1549.721$ $\mathrm{nm}$ (red line) and $\lambda_{1 \mathrm{D}}=1549.871$ (black line) $\mathrm{nm}$.

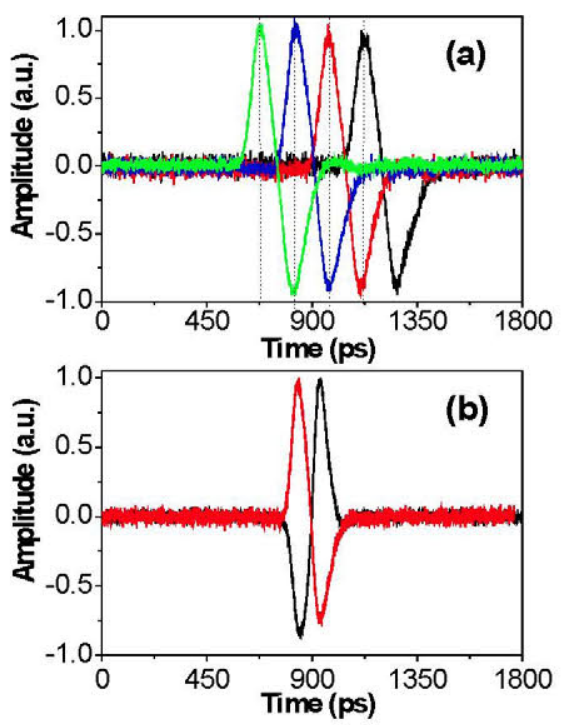

Fig. 3: BPM and PPM modulation for the generated UWB pulses. (a) PPM with a separation of $77 \mathrm{ps}$ (b) For BPM, original pulse (red line) and inverted pulse (black line).

\section{Conclusions}

We have proposed an efficient, reconfigurable and scalable UWB generator based on a customized photonic superstructure. Feasibility of this concept was experimentally proved by the generation of an UWB triplet pulse. Results reveal an improvement in terms of spectral efficiency from $23 \%$ to $65 \%$ when contrasting the base lower order doublet employed and the generated UWB triplet. Parallel to this, the system portraits an interesting flexibility to perform standard modulation formats such as BPM and PPM.

\section{Acknowledgements}

The research leading to these results has been funded by the national project TEC2010-21303-C0402 and TEC2011-26642 funded by the Ministerio de Economía y Competitividad and the projects FEDER UPVOV08-3E-008 and UPVOV10-3E-49.

\section{References}

[1] G.R. Aiello et al., "Ultra-wideband wireless systems," IEEE Microw. Mag., Vol. 4, no. 2, p. 36 (2003).

[2] Fed. Commun. Commission, "Revision of part 15 of the Commission's Rules Regarding Ultra-Wideband Transmission Systems," Tech. Rep., ET-Docket 98-153, no. 7 , p. FCCO2-48 (2002).

[3] S. Pan et al, "UWB-Over-Fiber Communications: Modulation and Transmission," J. Lightwave Technol., Vol. 28, no. 16, p. 2445 (2010).

[4] J. Capmany et al , "Microwave Photonic Signal Processing," J. Lightwave Technol., Vol. 31, no. 4, p. 571 (2013)

[5] Hongsan Sheng et al, "On the spectral and power requirements for ultra-wideband transmission," IEEE International Conference on Communications, Alaska, USA (2003).

[6] C. Wang et al., "All-fiber UWB pulse generation based on spectral shaping and dispersion-induced frequencyto-time conversion," IEEE Photon. Technol. Lett. Vol. 19, no. 3, p. 137 (2007).

[7] M. Bolea et al., "Optical UWB pulse generator using an $\mathrm{N}$ tap microwave photonic filter and phase inversion adaptable to different pulse modulation formats," Opt. Express., Vol. 17,no. 7, p. 5023 (2009).

[8] $\mathrm{H}$. Feng et al., "Photonic generation of reconfigurable orders ultrawideband signals by cascaded RSOAs," IEEE Photon. Technol. Lett., Vol. 26, no. 9, p. 908 (2014).

[9] S.T. Abraha et al., "Power-efficient impulse radio ultrawideband pulse generator based on the linear sum of modified doublet pulses," Opt. Lett., Vol. 36, no. 12, p. 2363 (2011).

[10]S. Tesfay et al., "Performance Evaluation of IR-UWB in short range fiber communication using linear combination of monocycles," J. Lightwave Technol., Vol. 29, no. 8, p. 1143 (2011).

[11]F.Zeng et al, "Ultrawideband Impulse Radio Signal Generation Using a High-Speed Electrooptic Phase Modulator and a Fiber-Bragg-Grating-Based Frequency Discriminator," IEEE Photon. Technol. Lett., Vol. 18, no. 19, p. 2062 (2006)

[12]M. Abtahi, M. Mirshafiei, J. Magné and A. Rusch, "Ultrawideband waveform generator based on optical pulseshaping and FBG tuning," IEEE Photon. Technol. Lett., vol. 20, no. 2, p. 135-137 (2008) 